\title{
Modeling of substorms and flares by the fast reconnection mechanism
}

\author{
M. Ugai, K. Kondoh, and T. Shimizu \\ Research Center for Space and Cosmic Evolution, Ehime University, Matsuyama 790-8577, Japan
}

(Received October 2, 2007; Revised March 29, 2008; Accepted June 4, 2008; Online published May 29, 2009)

\begin{abstract}
The 3D spontaneous fast reconnection model is applied to well-known signatures of geomagnetic substorms and solar flares. First, it is applied to the traveling compression regions (TCRs) associated with plasmoids propagating down the tail plasma sheet, known as a definite signature of geomagnetic substorms, and the in-situ satellite observations can be precisely explained, both qualitatively and quantitatively. Then, it is demonstrated that the magnetospheric current wedge drastically evolves through field-aligned currents to link the tail current to the auroral electrojet. It is also found that the well-known morphological features of two-ribbon flares can be explained by the fast reconnection model. In particular, the joule heating, associated with the flare current wedge, is shown to be important for the two-ribbon heating. Therefore, it is suggested that both solar flares and geomagnetic substorms result from the same physical mechanism, i.e., the fast reconnection mechanism.
\end{abstract}

Key words: Fast reconnection, substorms, flares, TCR, current wedge.

The fast reconnection mechanism involving standing slow shocks should be most responsible for explosive events observed in space plasmas (Shibata, 1999). Then, question is how the fast reconnection mechanism is realized in space plasmas of extremely large magnetic Reynolds number. A possible fast reconnection configuration was first proposed by Petschek (1964), and it was suggested that the fast reconnection mechanism may be determined by external boundary conditions (Vasyliunas, 1975). However, it can be realized only when a localized resistivity is applied (Ugai and Tsuda, 1977), and we have proposed the spontaneous fast reconnection model and demonstrated by 2D and 3D MHD simulations that the fast reconnection mechanism can be realized as an eventual solution by the nonlinear instability due to positive feedback between current-driven anomalous resistivities and global reconnection flows (Ugai, 1984, 1986, 1992, 1999; Ugai and Zheng, 2005).

In general, the energy conversion principle can directly be derived from Maxwell Equations as

$$
\frac{\partial}{\partial t}\left(\frac{\mathbf{B}^{2}}{2 \mu_{0}}+\frac{\epsilon_{0} \mathbf{E}^{2}}{2}\right)=-\nabla \cdot(\mathbf{E} \times \mathbf{B}) / \mu_{0}-\mathbf{E} \cdot \mathbf{J}
$$

The electric energy is negligible for the Alfven time scale, so that $\mathbf{E} \times \mathbf{B}$ indicates the magnetic energy flow, and $\mathbf{E} \cdot \mathbf{J}$ the rate of conversion between magnetic and plasma energies. Any fast reconnection process involves magnetic energy inflow $\mathbf{E} \times \mathbf{B}$ toward the diffusion region and hence requires the dissipation mechanism $\mathbf{E} \cdot \mathbf{J}>0$ in the diffusion (reconnection) region. Hence, the reconnection electric field $\mathbf{E}$ at the $\mathrm{X}$ reconnection point must be sustained by some dissipation mechanism. If there is no dissipation mechanism, the reconnection electric field should readily

Copyright (c) The Society of Geomagnetism and Earth, Planetary and Space Sciences (SGEPSS); The Seismological Society of Japan; The Volcanological Society of Japan; The Geodetic Society of Japan; The Japanese Society for Planetary Sciences; TERRAPUB be short-circuited by electrons in the small diffusion region of electron inertial length of $c / \omega_{\text {pe }}$.

The generalized Ohm's law may be written as $\mathbf{E}+\mathbf{u} \times \mathbf{B}=\mathbf{R}$, where $\mathbf{R}$ is composed of the resistivity term $\mathbf{R}_{\eta}$, the Hall term $\mathbf{R}_{\mathrm{H}}$, the electron pressure-tensor term $\mathbf{R}_{\mathrm{p}}$, and the electron inertial term $\mathbf{R}_{\mathrm{i}}$. For the Hall term, $\mathbf{R}_{\mathrm{H}} \cdot \mathbf{J}=0$, so that it cannot have any direct influence on the reconnection process, since it has no dissipation mechanism. In the diffusion region $(\mathbf{u} \times \mathbf{B} \sim 0)$, $\mathbf{R}$ can be described as $\eta \mathbf{J}$ since $\mathbf{E} \cdot \mathbf{J}>0$, where $\eta(>0)$ is the effective resistivity. The effective resistivity, usually provided by Coulomb collisions, is extremely small in space plasmas, and if current densities are notably intensified, it may result from wave-particle collisions due to current-driven microinstabilites, such as ion acoustic, two-stream (Buneman), lower-hybrid drift instabilities (Lui, 2001). In fact, anomalous resistivities are detected in laboratory plasmas (Ono et al., 2001).

First, let us examine the physical conditions for the fast reconnection evolution to be realized in actual $3 \mathrm{D}$ systems. Initially, we assume a current sheet system that contains antiparallel magnetic fields $B_{x}$ and a sheet current in $|y|<1$. Initiated by a small disturbance, all the phenomena grow by the self-consistent interaction between the reconnection flow and the effective resistivity. In order to examine how magnetic reconnection is influenced by the effective resistivity, we may assume the following resistivity models. For the current-driven anomalous resistivity model (A), $\eta(\mathbf{r}, t)=k_{\mathrm{R}}\left[V_{\mathrm{d}}(\mathbf{r}, t)-V_{\mathrm{C}}\right]$ for $V_{\mathrm{d}}>V_{\mathrm{C}}$ $\left(V_{\mathrm{d}}=|\mathbf{J} / \rho|\right)$, where $\rho$ is plasma density, and $V_{\mathrm{d}}$ the relative electron-ion drift velocity. For another anomalous resistivity model (B), $\eta(\mathbf{r}, t)=k_{\mathrm{J}}\left[|\mathbf{J}(\mathbf{r}, t)|-J_{\mathrm{C}}\right]$ for $|\mathbf{J}|>J_{\mathrm{C}}$. For the model $(\mathrm{C})$, the Spitzer resistivity is assumed as, $\eta(\mathbf{r}, t)=k_{\mathrm{C}}\left[T(\mathbf{r}, t) / T_{0}\right]^{-3 / 2}$, where $T$ is the temperature.

We find that for the anomalous resistivity model (A) or (B), if the threshold $V_{\mathrm{C}}$ or $J_{\mathrm{C}}$ is sufficiently large, the fast 


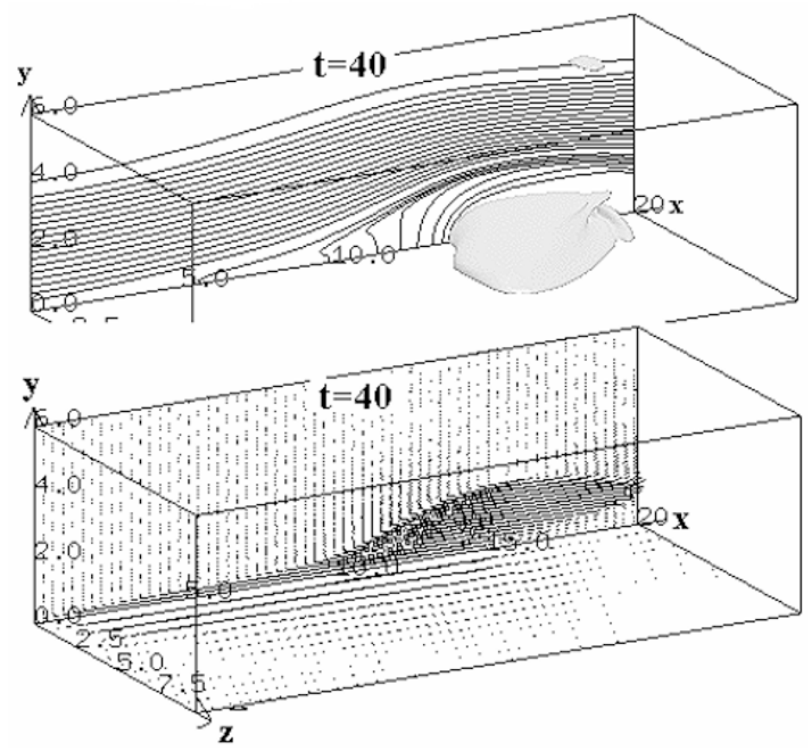

Fig. 1. (Upper) Magnetic field configuration with the isosurface of the plasma pressure $P$, and (lower) plasma flow vectors for the anomalous resistivity model (A), where the $\mathrm{X}$ neutral point is located at the origin.

reconnection mechanism fully evolves as a nonlinear instability because of the positive feedback, since the reconnection flows grow so as to enhance the current density at the X point by the pinch effect (Ugai, 1984, 1986, 1999). Figure 1 typically shows the resulting configuration for the anomalous resistivity model (A), where the fast reconnection mechanism involving slow shock builds up, and a largescale plasmoid is formed ahead of the Alfvenic fast reconnection jet. Here, the simulation domain is a rectangular box, $0 \leq x \leq L_{x}=20,0 \leq y \leq L_{y}=6$, and $0 \leq z \leq$ $L_{z}=9.8$, with the mesh sizes $\triangle x=0.04, \triangle y=0.015$ and $\triangle z=0.1$, and the conventional symmetry boundary conditions are assumed on the $z=0, x=0$, and $y=0$ planes with the other outer planes being free boundaries. For the Spitzer resistivity model (C), no effective reconnection occurs because of the negative feedback, since the resistivity becomes reduced because of the increase in temperature $T$ in the diffusion region (Ugai and Zheng, 2005). Note that magnetic reconnection is strongly influenced by the effective resistivity, and the fast reconnection mechanism can be realized for current-driven anomalous resistivities even in general three dimensions.

Next, let us apply the spontaneous fast reconnection model to substorm signatures observed by satellites. The so-called traveling compression regions (TCRs) have been clearly observed in the tail lobe of the Earth's magnetosphere, and they were qualitatively expected to result from the plasmoid bulge propagating down the central plasma sheet. The TCR signatures have been studied in detail (Slavin et al., 1993), and Fig. 2 typically shows the observed magnetic field variations, which indicates the pulselike compression of the earthward $\left(B_{x}\right)$ field component as well as the northward to the southward tilting of the $B_{z}$ field component. These fundamental features of TCR have been theoretically discussed by Birn (1992) and by Young and Hameiri (1992), but have not been verified in detail by nu-

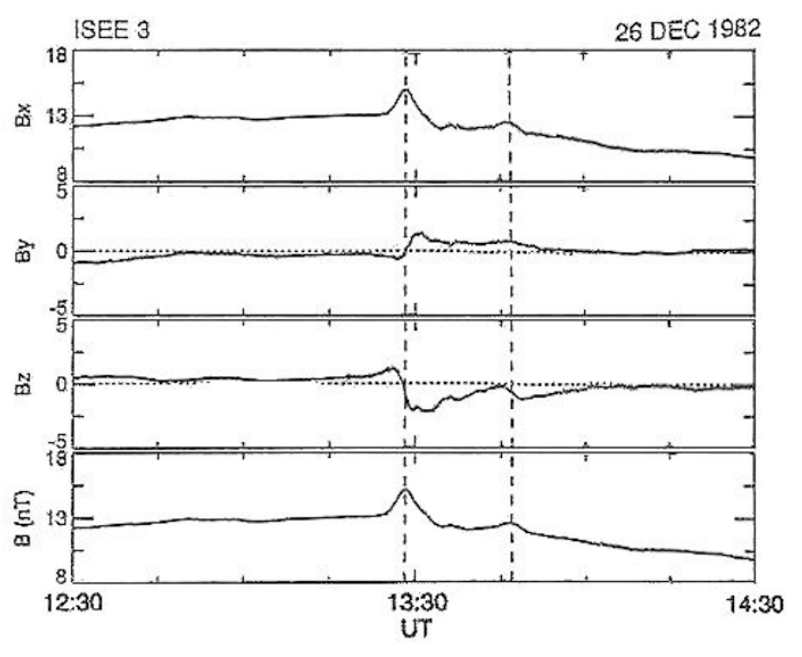

Fig. 2. ISEE 3 magnetic field observations taken in the north lobe of the tail at $x=-73 R_{\mathrm{e}}$ in GSM coordinates (after Slavin et al., 1993).

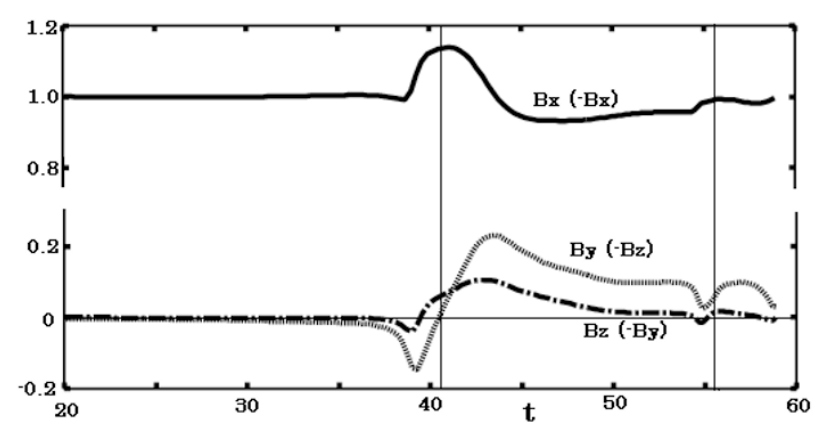

Fig. 3. Temporal variations of magnetic fields observed by virtual satellites located in the magnetic field (low- $\beta$ ) region, where $B_{x}, B_{y}$ and $B_{z}$ correspond to $-B_{x},-B_{z}$ and $-B_{y}$ in GSM coordinates, respectively.

\section{merical simulations.}

In order to clarify the physical mechanism of TCR, 3D MHD simulations of the spontaneous fast reconnection model are performed with the same simulation model as in Fig. 1. It should be noted that unlike the traditional plasmoid, the resulting plasmoid contains no closed field lines and is caused and sustained by the Alfvenic fast reconnection jet. Then, in order to directly compare the simulation results with the satellite observations, virtual satellites are located in the magnetic field region outside the central plasma sheet in the simulation domain, which can readily measure the temporal variations of magnetic fields in accordance with the plasmoid propagation (Ugai and Zheng, 2006a). Figure 3 shows the field components, measured by the virtual satellites, which indicate the pulse-like field compression $B_{x}$ with the bipolar changes in $B_{y}$ and $B_{z}$. Then, another tearing suddenly occurs, leading to a small plasmoid propagating outward, so that a small TCR is observed at $t \sim 56$. These results are in good agreement with the actual satellite observations (Fig. 2) (Ugai and Zheng, 2006b).

Other well-known substorm signatures are the earthward jet and the magnetic field dipolarization. In particular, when a substorm builds up, the tail current sheet may suddenly 

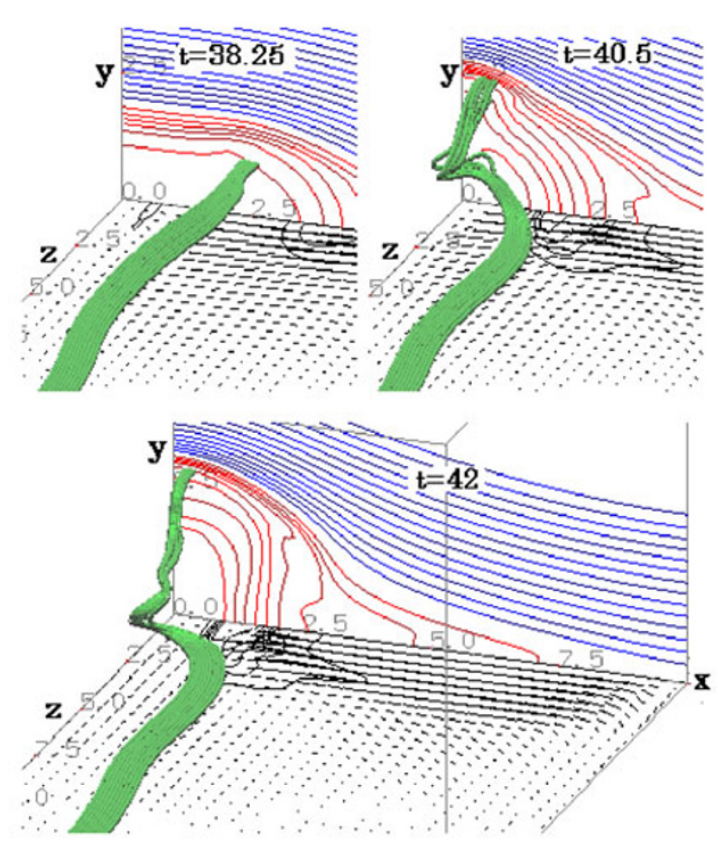

Fig. 4. (Color online) Current flow lines starting from the segment, $2.16<x<2.72$ at $y=0.27$ and $z=13$, at different times, where contour lines of the reconnected field component $B_{y}$ and the flow velocity vectors $\mathbf{u}$ in the $(z, x)$ plane are also shown, where the $\mathrm{X}$ neutral point is located at $x=L_{x}=10$.

be disrupted so that a substorm current wedge evolves to link the tail current to the auroral electrojet through fieldaligned currents (McPherron et al., 1973). The importance of current wedge has widely been recognized by satellite and ground observations, but this long-standing question has never been resolved theoretically. Recently, it is demonstrated on the basis of the 3D spontaneous fast reconnection model that when the fast reconnection jet collides with the closed field lines in the near-Earth magnetosphere, the drastic current wedge evolution can be realized (Ugai and Kondoh, 2006).

We have also found that only when the east-west width of the tail current sheet is 3-4 times larger than its thickness, the 3D fast reconnection mechanism and the current wedge can fully be set up (Ugai and Shimizu, 1996; Ugai, 2007). For this case, Fig. 4 shows the resulting current flow lines, where the contour lines of the reconnected field component $B_{y}$ and the flow velocity vectors $\mathbf{u}$ in the $(z, x)$ plane are also shown. In this model, magnetic reconnection is initiated at $x=L_{x}=10$, and the $x=10$ plane as well as the $x=0$ plane is assumed to be the symmetry boundary. We readily see that when the closed field lines in the near-Earth magnetosphere is compressed by the 3D fast reconnection jet, the sheet current $\left(J_{z}<0\right)$ ahead of the closed field lines drastically turns its direction toward the foot points of close field lines through field-aligned currents. This is generally consistent with the predicted substorm current wedge.

In view of morphological similarities between flares and substorms, the spontaneous fast reconnection model may also be applied to two-ribbon flares. Unlike in-situ observations in the Earth's magnetosphere, magnetic fields can hardly be measured in the solar corona. In this sense, any theoretical model of solar flare is circumstantial, so that it is
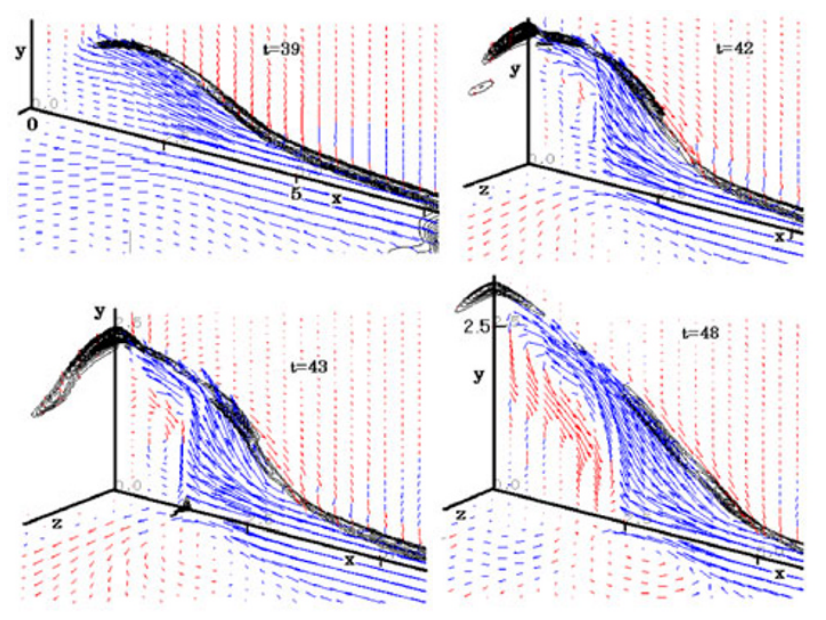

Fig. 5. (Color online) Plasma flow vectors and contour lines of the joule heating $\eta \mathbf{J}^{2}$, where the fast reconnection flow velocity $u_{x}<0$ attains the Alfven velocity, $V_{\mathrm{A}} \sim-2.7$, measured in the low- $\beta$ region.

essential to clarify the well-known morphological features that have been obtained from satellite observations. The previous theories were mostly based on the cartoon model based on the 2D Petschek reconnection configuration, and it was supposed that the chromospheric flare heating should be caused directly from the coronal plasma heated and accelerated by $2 \mathrm{D}$ reconnection shocks.

The solar surface is modeled as follows. An antiparallel magnetic field $\mathbf{B}=\left[B_{x}(y), 0,0\right]$ is initially assumed as: $B_{x}(y)=\sin (\pi y / 2)$ for $0<y<1 ; B_{x}=1$ for $1<y<4$; $B_{x}=\cos [(y-4) \pi / 1.2]$ for $4<y<4.6 ; B_{x}=0$ for $y>$ 4.6; also, $B_{x}(y)=-B_{x}(-y)$ for $y<0$. Fluid velocity $\mathbf{u}=$ $(0,0,0)$, and plasma pressure $P(y)$ satisfies the pressurebalance condition, $P+B_{x}^{2}=1+\beta_{0}$, where $\beta_{0}=0.15$ is taken. Also, plasma density $\rho$ initially satisfies $\rho(x, y)=$ $\left[R_{0} \exp \left[-(x / 0.4)^{4}\right]+1\right] P(y) /\left(1+\beta_{0}\right)$, where $R_{0}=100$ is taken, so that $x=0$ corresponds to the chromosphere, and the corona is for $x \gg 0.4$. In the chromosphere, placed in the $x=0$ plane, the plasma density is assumed to be 100 times larger than in the corona for $x>0.4$, so that the temperature in the corona is 100 times larger than in the chromosphere.

An initial disturbance is imposed at $x=L_{x}=10$ on the initial configuration, and then the current-driven anomalous resistivity models are assumed. Figure 5 illustrates the resulting plasma flow vectors and contour lines of the joule heating $\eta \mathbf{J}^{2}$. The joule heating in the $(x, y)$ plane mostly indicates the standing slow shock layer, across which the plasma is accelerated to the Alfven velocity. The plasmoid, propagating in the negative $x$ direction (at $t=39$ ), collides with the chromosphere $(x=0)$ at $t \sim 42$, leading to sudden current wedge evolution, which may be called the flare current wedge by analogy with the substrom current wedge. For $t>42$, distinct plasma downflows $\left(u_{x}<0\right)$ toward the chromosphere $(x=0)$ occur along the magnetic loop boundary.

The flare current wedge causes an extreme increase in the current density (hence an anomalous resistivity) in the chromosphere $(x=0)$. Hence, Fig. 5 indicates that re- 


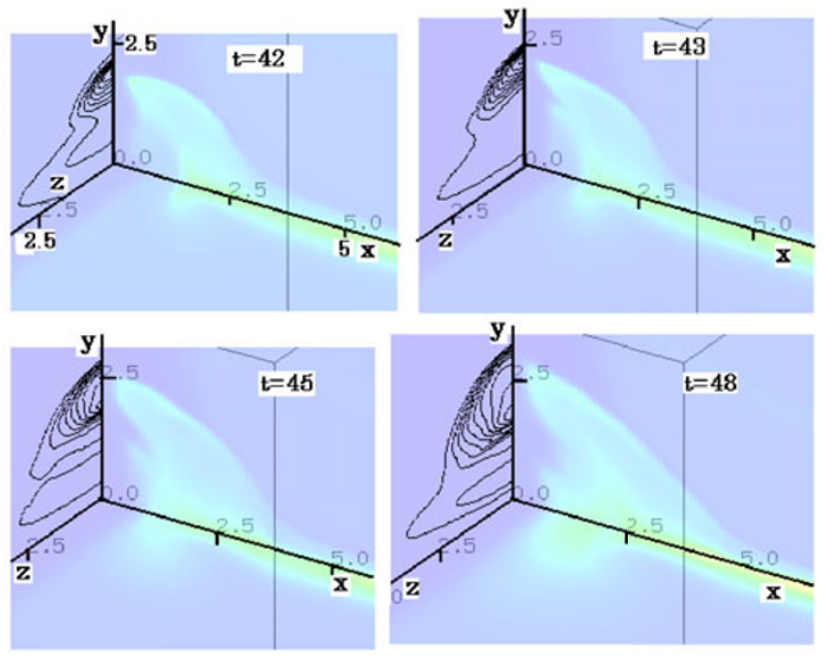

Fig. 6. (Color online) Temperature distributions, shown by contour lines at $x=0$, where the maximum temperature becomes more than 20 times its initial value.

markable joule heating occurs along the thin layer in the chromosphere near the seperatrix, which bounds the preand post-reconnection field lines. In particular, the strongest heating occurs impulsively at times $t=42$ and 43. Accordingly, Fig. 6 shows the resulting temperature distributions, and demonstrates that at $t=42$ the temperature is suddenly enhanced distinctly in the chromospheric thin layer, which presents the two-ribbon structure because of the symmetry boundary conditions. Hence, the plasma pressure is notably enhanced in the chromosphere to cause distinct upward flows $\left(u_{x}>0\right)$ from the chromosphere at $t=48$ inside the loop, which is consistent with the so-called chromospheric evaporation observed during flares. The joule heating layer moves in the positive $y$ direction as the reconnected field lines are piled up, and the cusp-shaped flare loop expands outward. These results are consistent with the well-known morphological features of two-ribbon flares.

In summary, in-situ satellite observations of TCR signatures can exactly be explained by the 3D spontaneous fast reconnection model (Figs. 2 and 3). Also, the 3D fast reconnection jet causes drastic evolution of magnetospheric current wedge (Fig. 4). For this theoretical model, currentdriven anomalous resistivities are essential, and the current disruption occurs just ahead of the closed field lines to form the current wedge (Fig. 4). Interestingly, these basic features are rather consistent with those of the current disruption model (Lui, 1996). In addition, it is demonstrated that the well-known morphological features of two-ribbon flares are pertinently explained by this theoretical model (Figs. 5 and 6). In particular, the drastic increase in the chromospheric temperature occurs in the shape of two ribbons, and the resulting coronal flare loop is expanding outward. Also, the definite chromospheric evaporation occurs. These re- sults suggest that both solar flares and geomagnetic substorms result from the same physical mechanism, i.e., the fast reconnection mechanism.

Acknowledgments. This work was supported by Grant-in-Aids for Creative Scientific Research 'The Basic Study of Space Weather Prediction' (17GS0208, Head Investigator: K. Shibata) from the Ministry of Education in Japan, Mitsubishi Foundation, RISH of Kyoto University, and Solar-Terrestrial Environment Laboratory of Nagoya University.

\section{References}

Birn, J., Quasi-steady current sheet structures with field-aligned flow, $J$. Geophys. Res., 97, 16,817-16,826, 1992.

Lui, A. T. Y., Current disruption in the Earth's magnetosphere: observations and models, J. Geophys. Res., 101, 13067-13088, 1996.

Lui, A. T. Y., A multiscale model for substorms, Space Sci. Rev., 95, 325345, 2001.

McPherron, R. L., C. T. Russel, and M. P. Aurbry, Satellite studies of magnetospheric substorms on August 15, 1968. 9. Phenomenological model for substorms, J. Geophys. Res., 78, 3131-3149, 1973.

Ono, Y. et al., Fast compression of a current sheet during externally driven magnetic reconnection, Earth Planets Space, 53, 521-526, 2001.

Petshcek, H. E., Magnetic field annihilation, in AAS-NASA Symposium on the Physics of Solar Flares, NASA Spec. Pub., SP-50, 425-439, 1964.

Shibata, K., Evidence of magnetic reconnection in solar flares and a unified model of flares, Astrophys. Space Sci., 264, 129-144, 1999.

Slavin, J. A. et al., ISEE 3 observations of traveling compression regions in the Earth's magnetotail, J. Geophys. Res., 98, 15425-15446, 1993.

Ugai, M., Self-consistent development of fast magnetic reconnection with anomalous plasma resistivity, Plasma Phys. Controlled Fusion, 26, 1549-1563, 1984

Ugai, M., Global dynamics and rapid collapse of an isolated current-sheet system enclosed by free boundaries, Phys. Fluids, 29, 3659-3667, 1986.

Ugai, M., Computer studies on development of the fast reconnection mechanism for different resistivity models, Phys. Fluids B, 4, 2953-2963, 1992.

Ugai, M., Computer studies on the spontaneous fast reconnection model as a nonlinear instability, Phys. Plasmas, 6, 1522-1531, 1999.

Ugai, M., Conditions for drastic evolution of magnetospheric current wedge, Phys. Plasmas, 14, 062902, 2007.

Ugai, M. and T. Tsuda, Magnetic field-line reconnexion by localized enhancement of resistivity. Part 1. Evolution in a compressible MHD fluid, J. Plasma Phys., 17, 337-356, 1977.

Ugai, M. and T. Shimizu, Computer studies on the spontaneous fast reconnection mechanism in three dimensions, Phys. Plasmas, 3, 853-862, 1996.

Ugai, M. and L. Zheng, Conditions for the fast reconnection mechanism in three dimensions, Phys. Plasmas, 12, 092312, 2005.

Ugai, M. and L. Zheng, Modeling of traveling compression regions in the Earth's magnetotail by the spontaneous fast reconnection model, Phys. Plasmas, 3, 032901, 2006a.

Ugai, M. and L. Zheng, Parametric studies on traveling compression regions observed in the Earth's magnetotail, Phys. Plasmas, 13, 062906, 2006 b.

Ugai, M. and K. Kondoh, Evolution of magnetospheric current wedge by the spontaneous fast reconnection model, Phys. Plasmas, 13, 102903, 2006.

Vasyliunas, V. M., Theoretical models of magnetic field line merging, Rev. Geophys. Space Phys., 13, 303-336, 1975.

Young, R. and E. Hameiri, Approximate magnetotail equilibria with parallel flow, J. Geophys. Res., 97, 16789-16802, 1992.

M. Ugai (e-mail: ugai@cosmos.ehime-u.ac.jp), K. Kondoh, and T. Shimizu 